\title{
Estrategia para la retención en carrera de ingeniería: Ingeniería Electrónica de la Universidad Nacional de Tucumán
}

\author{
Retention strategy in engineering career: Electronic Engineering of the Tucumán \\ National University
}

\author{
María de los A. Gómez López ${ }^{\# 1}$, Carlos Sueldo ${ }^{\# 2}$, Leonardo Del Sancio \#3 \\ \# Dpto. Electricidad, Electrónica y Computación, Universidad Nacional de Tucumán \\ San Miguel de Tucumán, Argentina \\ 1 mgomezlopez@herrera.unt.edu.ar \\ ${ }^{2}$ csueldodherrera.unt.edu.ar \\ 3 Idelsancio@herrera.unt.edu.ar
}

Recibido: 16/02/21; Aceptado: 28/03/21

\begin{abstract}
This paper aims at presenting a retention strategy used by the Electronic Engineer career from the Universidad Nacional de Tucumán in order to motivate, integrate and keep their students. The procedure used is the implementation of practical workshops aimed at students of all years of the career who have as premise "to learn by doing". The workshops seek to bring the student in all aspects of the exercise of the profession. They are taught one at a time in sequence and are assessed by completing a simple project. The results of the procedure used were measured by the number of enrolled students and surveys verifying satisfaction
\end{abstract}

Keywords: Retention strategy; Dropout; Electronic Engineer.

Resumen - Este trabajo tiene como objetivo presentar una estrategia de retención empleada por la carrera de Ingeniería Electrónica de la Universidad Nacional de Tucumán como una herramienta para motivar, integrar y retener a sus estudiantes. Se emplea como procedimiento la implementación de talleres prácticos orientados a estudiantes de todos los años de la carrera que tienen como premisa "aprender haciendo". Buscan acercar al estudiante en aspectos propios del ejercicio de la profesión. Se imparten de uno en uno en forma secuencial y se evalúan mediante la realización de un proyecto simple. Los resultados de la metodología empleada se midieron mediante el número de estudiantes inscriptos $y$ encuestas comprobando la satisfacción.

Palabras clave: Estrategia de retención; Abandono; Ingeniería Electrónica.

\section{INTRODUCCION}

La meta fundamental de un sistema educativo eficiente es la culminación de los procesos académicos con éxito, disminuir las cifras de abandono, de deserción y lograr la permanencia. La evidencia indica que la deserción universitaria es un fenómeno global con implicaciones y retroceso en lo económico, académico, social y profesional [1]. El trabajo propone una estrategia académica de la carrera de Ingeniería Electrónica de la Facultad de Ciencias
Exactas y Tecnología (FACET) de la Universidad Nacional de Tucumán (UNT), con el fin de fortalecer la permanencia, mejorar la visibilidad tanto interna como externa a la Unidad Académica (UA) [2], aumentar los índices de matriculación, mejorar la motivación de los estudiantes actuales y finalmente, fomentar actividades extracurriculares acordes a alumnos de todos los años del Plan de Estudio [3], como una manera de acercarlos al ejercicio de la profesión.

Los índices de matriculación en las diferentes ingenierías en la UNT vienen bajando año a año. Esta realidad no es ajena a la que viven otras instituciones públicas de Educación Superior en el país. Ya el "Plan estratégico de formación de ingenieros 2012-2016" de la República Argentina [4] contempla esta realidad. Plantea incrementar la cantidad de graduados en ingeniería en un $100 \%$ en 2021 con respecto al 2009 en carreras acreditadas en su segundo ciclo de evaluación. Para ello, explicita claramente objetivos como a) incrementar la retención en el ciclo básico; b) incrementar la retención en el ciclo de especialización; y c) incrementar la graduación de alumnos avanzados. La carrera de Ingeniería Electrónica de la UNT, en los últimos años, viene haciendo significativos esfuerzos para atender esta coyuntura nacional de las instituciones universitarias. En este sentido, está implementando un nuevo plan de estudios basado en educación por competencias con centro en el estudiante. Este plan se concibe como la combinación de habilidades, actitudes, destrezas y conocimientos del estudiante, así como la inclusión de la disposición para aprender [5].

En este contexto, como medida inmediata de retención y motivación de estudiantes de Ingeniería Electrónica, la Comisión Académica (CA) de la carrera implementó, por primera vez, durante el año 2019 un conjunto de talleres prácticos específicos en los distintos ejes temáticos, con una aceptación satisfactoria de los estudiantes medida a través de la matriculación en los mismos. Estos talleres fueron 
llamados INGETRÓNICA y debido a la premisa de "aprender haciendo" se imparten en los laboratorios de formación experimental de la carrera. Pretenden que el estudiante complemente su formación principalmente desde la instuición y la práctica experimental.

Además de su rol estratégico para mejorar la retención y motivación, los talleres persiguen el objetivo de integrar a los estudiantes de los diferentes años considerándolos como pares durante la ejecución de los mismos. Paralelamente, también se impacta positivamente en la vinculación de los egresados con la carrera. Esta articulación se logra invitando a los egresados en ejercicios de la profesión y expertos en áreas específicas para que impartan los talleres. De esta forma, no sólo se consigue aprovechar la experiencia profesional de los mismos, sino que también los estudiantes tienen la posibilidad de formarse en aquellos aspectos propios de la profesión.

Los cursos se destinan principalmente a todos los estudiantes de Ingeniería Electrónica, pero se admiten también a participantes de ingenierías a fines a las áreas electrónica, computación, informática y bioingeniería. Se distribuyen en jornadas de cuatro horas una vez por semana, los días viernes a la tarde o sábados por la mañana para que al estudiante no tenga superposición de horarios con las materias obligatorias de la currícula.

La carrera pretende mantener en el tiempo estos espacios de formación complementaria y actualmente lleva a cabo la segunda edición de INGETRÓNICA.

\section{SITUACIÓN ACTUAL}

Actualmente, la UNT tiene un destacado rol social y debe adecuarse a los tiempos que le toca vivir. Debe preparar a sus egresados para afrontar un mundo de gran complejidad, alta competitividad y una fuerte acentuación del individualismo [6][7].

En consonancia con la función de extensión de la UNT cuya misión es formular y ejecutar políticas y acciones que promuevan el diálogo permanente de la UNT con la sociedad, tendientes al aporte de soluciones a las problemáticas prioritarias de nuestra región (Resolución Rectoral de la UNT 0004/2018), la carrera tiene una misión claramente definida.

1) Misión: Persigue la formulación de sus propias políticas $\mathrm{y}$ acciones tendientes a aumentar el número ingenieros electrónicos graduados en la región. En este marco, la carrera formula y ejecuta políticas de grado, consecuentes con el fortalecimiento de la calidad académica de los graduados. Estas políticas y acciones se resumen en desarrollar espacios de coordinación y gestión académica que permitan trazar políticas transversales; promover y desarrollar actividades que permitan divulgar, publicar e intercambiar experiencias, investigaciones y políticas relativas al ámbito de su competencia; impulsar políticas de ingreso, permanencia y graduación de los/as estudiantes; procurar la formación continua de los/as docentes tanto disciplinar como pedagógica; y finalmente, promover la vinculación activa con los/as egresados/as de la carrera, impulsando su participación en actividades académicas, científicas y de extensión.

2) Fortalezas de la carrera: Cuenta con el personal docente, la infraestructura y la organización suficiente para la concreción de la mayor parte de sus funciones. Cuenta con una favorable relación docente-alumno para el desarrollo de la enseñanza. Ha sostenido la matrícula a lo largo de los últimos 8 años. Desarrolla estrategias para afrontar los problemas de retención y duración de la carrera, particularmente, el abandono en los dos primeros años del Plan de Estudio.

3) Déficits de la carrera: Cuenta con problemas de retención durante los dos primeros años de cursado, una excesiva duración y baja tasa de graduación. Se puede afirmar también que los vínculos de la carrera con el sector socio productivo son insuficientes. Finalmente, no ofrece la modalidad de cursado a distancia lo cual limita la demanda académica considerablemente.

4) Oportunidades de mejoramiento: Unas de las más importantes es fortalecer prácticas de articulación docentealumno entre la investigación y extensión. Organizar actividades extracurriculares con estudiantes de todos los años de la carrera. Fortalecer el vínculo con los graduados también resulta en una oportunidad si se quiere mejorar el vínculo con el sector socio-productivo.

En este contexto, la carrera se propone mejorar los índices de retención creando actividades que motiven a los estudiantes y los acerque a la disciplina. Se espera poner en funcionamiento un sistema de acompañamiento a las trayectorias estudiantiles en la carrera. Para mejorar la vinculación con el sector socioproductivo se propone generar los puentes con el mercado laboral generando convenios y alianzas con sectores referentes de la región.

Se hace notal que para cumplir con estos objetivos, la carrera cuenta con RRHH e infraestructura suficiente. Cuenta con un esquema organizacional encabezado por la Secretaría Académica que formula las políticas y estrategias marcos de la FACET dejando atribuciones a las carreras para plantear las medidas particulares.

\section{METODOLOGÍA}

Se elabora un procedimiento con el fin de facilitar las tareas organizativas de los talleres. El procedimiento describe la manera de difundir y comunicar, una sugerencia del cronograma y la manera de realizar la preinscripción e inscripción final.

\section{A. Difusión y comunicación}

Se emplean las redes sociales de divulgación de la carrera, Instagram, Facebook y Twitter. Además, se emplean las vías de comunicación oficiales de la FACET de la UNT. Las publicaciones se realizan mediante secuencias de imágenes cuidadosamente elaboradas para que resulten atractivas y novedosas. En ellas se dan a conocer todos los aspectos generales y específicos de los talleres.

\section{B. Esquema de organización}

Los cursos se organizan en 4 etapas:

1) Definición: Es esta etapa se interactúa con los disertantes para la especificación de la temática y el tiempo estimado de cada taller. La CA arma una base de datos de posibles disertantes expertos con egresados externos a la UA en ejercicio de la profesión y con egresados docentes de la UA. 
La CA los contacta vía email y les hace la invitación para participar. Se les solicita, en caso de estar interesados, que realicen una propuesta formal con una temática específica, siguiendo una plantilla predeterminada. En esta plantilla se describen aspectos como título, fundamentación, objetivos, contenidos mínimos, metodología para el aprendizaje y duración estimada. Junto con la propuesta, el disertante debe presentar su currículum vitae especificando, principalmente, su experiencia en el ejercicio de la profesión.

De todas las propuestas recibidas, la $\mathrm{CA}$ hace una selección de aquellas que son viables y que considera que agregan valor en la formación del ingeniero electrónico.

Con toda esta información se elabora el cronograma académico de los talleres, consensuado con los disertantes, dictando siempre de a uno y sin superposición. Este cronograma, junto a los detalles del taller y los currículums vitae de los disertantes se eleva inmediatamente a las autoridades de la UA para gestionar los permisos y avales correspondientes para el dictado

2) Preinscripción: Fijado el cronograma, se hace el lanzamiento del primer taller y se procede a implementar una preinscripción virtual por cupo y lista de espera. Para hacerla efectiva, se emplea como herramienta estandarizada Google Drive y Google Docs para implementar los formularios. En esta preinscripción, se demanda información sobre los datos personales del aspirante, medio por el cual le llegó la información y su condición actual de estudiante o no. Para el caso de estudiantes, se indaga carrera y año en curso. Para el caso de graduados, se indaga sobre el título de grado u otro y ocupación.

Esta etapa es importante ya que permite a la carrera crear una idea de la cantidad de alumnos interesados, el año que están cursando actualmente del Plan de Estudios, la carrera a la que pertenecen $\mathrm{y}$, sobre todo, la cantidad de recursos tanto de infraestructuras como de equipamiento que será necesario para el dictado del mismo.

3) Matriculación: Se abre inmediatamente después de cerrada la preinscripción. Se emplea el Campus Virtual de la FACET para tal fin. En este espacio, e incorporados como cursos de capacitación de Ingeniería Electrónica, se encuentra el aula virtual que aloja los talleres y que habilita sólo la actual para matriculación. El estudiante aquí puede acceder a todos los detalles del taller como título, disertante, día y horarios, lugar y materiales necesarios.

4) Ejecución y cierre: Una vez evaluado el taller, se cierra emitiendo la certificación correspondiente de aprobación o asistencia según sea el caso. Inmediatamente la carrera habilita la preinscripción del siguiente taller y el ciclo se concluye cuando todos los talleres han sido cerrados.

\section{Descripción de la muestra}

Se adopta una muestra de alumnos resultante de la etapa de inscripción final a los talleres. Los mecanismos de difusión mediante redes sociales se mostraron eficaces en esta primera edición. Esto trajo como consecuencia algunas singularidades en la muestra de estudiantes:

1) Alumnos: Está constituida por alumnos externos a la UA y por alumnos internos a la misma
2) Alumnos internos: Los estudiantes internos a la UA, no sólo son estudiantes de la carrera de ingeniería electrónica, sino que también lo son de otras ingenierías afines al área electrónica. Este es el caso de ingeniería biomédica, ingeniería en computación e ingeniería eléctrica.

3) Alumnos externos: Un significativo número de estudiantes de "otras carreras" de la UA, no afines a la electrónica, formaron parte de la muestra. Tal es el caso de ingeniería mecánica, ingeniería química, ingeniería industrial y licenciatura en física.

4) Muestra de alumnos: La muestra está constituida por alumnos graduados y no graduados. Los graduados son docentes y profesionales en ejercicio.

5) Alumnos docentes: El extracto de los docentes está integrado por los propios de la UA y externos a la misma. Los docentes externos se componen de docentes universitarios y de escuelas técnicas.

6) Alumnos no docentes: El extracto de los no docentes está integrado por profesionales en ejercicio de la profesión en empresas privadas

\section{DESARROLLO}

\section{A. Talleres}

La Tabla I describe la temática y la duración de cada taller [8]. La primera columna muestra su número de identificación, la segunda columna muestra su código, la tercera el nombre del taller y la cuarta su intervalo de duración. Se observa que en la primera edición se impartieron cinco talleres prácticos de los cuales sólo uno es del área de las ciencias sociales y hace a la formación complementaria de la carrera.

LA TABLA I

DURACIÓN Y CÓDIGOS DE LOS TALLERES DE INGENIERÍA ELECTRÓNICA DE LA FACET-UNT - PRIMERA EDICIÓN

\begin{tabular}{|l|l|l|l|}
\hline Nro & Código & \multicolumn{1}{|c|}{ Nombre del taller } & Período de ejecución \\
\hline 1 & $P A$ & Plataforma Arduino & 4 semanas \\
\hline 2 & $F O$ & $\begin{array}{l}\text { iPor qué la Fibra } \\
\text { Optica está de moda? }\end{array}$ & 2 semanas \\
\hline 3 & $P C B$ & $\begin{array}{l}\text { Diseño y desarrollo de } \\
\text { circuitos impresos }\end{array}$ & 4 semanas \\
\hline 4 & $P I C$ & $\begin{array}{l}\text { Programación de } \\
\text { Microcontroladores } \\
\text { PIC }\end{array}$ & 4 semanas \\
\hline 5 & $F C$ & $\begin{array}{l}\text { Comunicación } \\
\text { integral. Formación } \\
\text { complementaria }\end{array}$ & 1 semana \\
\hline
\end{tabular}

\section{B. Estadísticas}

\section{1) Distribución inscripciones por carrera}

La Tabla II describe la distribución por carrera de inscriptos. Los números de la segunda columna representan los porcentajes con respecto a la totalidad de inscriptos efectiva en todos los talleres. De la misma manera, los números de la tercera columna representan las cantidades absolutas de inscriptos a cada taller. 
TABLA II

PORCENTAJE DE PARTICIPANTES POR CARRERA DE LOS TALLERES DE INGENIERÍA ELECTRÓNICA DE LA FACET-UNT

\begin{tabular}{|l|l|l|}
\hline Carreras & $\begin{array}{c}\text { Inscripciones } \\
{[\%]}\end{array}$ & $\begin{array}{c}\text { Cantidad de } \\
\text { Inscripciones }\end{array}$ \\
\hline Ingeniería electrónica & 38 & 49 \\
\hline Ingeniería Biomédica & 27 & 36 \\
\hline Ingeniería en computación & 11 & 14 \\
\hline Ingeniería eléctrica & 4 & 5 \\
\hline Otras carreras & 5 & 7 \\
\hline Externas UA & 15 & 20 \\
\hline
\end{tabular}

La columna "Carreras" muestra las carreras internas y externas a la UA. Las cuatro filas correspondientes a las ingenierías junto a la denominada "Otras carreras" representan las carreras internas a la UA. "Otras carreras" incluye ingenierías como mecánica, química e industrial, así como también Licenciatura en Física. La fila titulada "Externas UA" representa a alumnos graduados y no graduados. Los primeros son profesionales que ejercen la profesión en empresas públicas o privadas y los segundos son estudiantes de grado de otras instituciones de Educación Superior tanto públicas como privadas.

2) Distribución por nivel de avance en la carrera de Ingeniería Electrónica

La Tabla III describe la distribución por nivel de avance (año de cursado) de inscriptos en la carrera de Ingeniería Electrónica que participaron de los cursos.

La Tabla IV describe la distribución por nivel de avance y por taller, de inscriptos de Ingeniería Electrónica expresada en porcentaje de los alumnos que tomaron cada uno de los talleres. Los números representan los porcentajes con respecto al número de inscriptos de Ingeniería Electrónica en cada taller. De esta forma las columnas suman $100 \%$.

\section{3) Distribución por carreras}

La Tabla V describe la distribución de inscriptos expresada en porcentaje de los alumnos que tomaron cada uno de los talleres según diferentes carreras.

La primera columna muestra dos tipos de carreras: internas y externas a la UA. Las cuatro filas correspondientes a las ingenierías junto a la denominada "Otras carreras" representan las internas a la UA. "Otras carreras" incluye ingenierías como mecánica, química e industrial, así como también Licenciatura en Física. La fila titulada "Externos UA" representa a alumnos graduados y no graduados. Los primeros son profesionales que ejercen la profesión en empresas públicas o privadas y los segundos son estudiantes de grado de otras instituciones de Educación Superior tanto públicas como privadas. Los números de la primera columna de cada taller representan los porcentajes con respecto al número de inscriptos finales en cada curso. De esta forma las columnas suman $100 \%$. En la segunda columna de cada taller, los números representan la cantidad de inscriptos absoluta el taller correspondiente.

\section{4) Distribución de inscripciones por talleres}

En la Tabla VI se muestra la distribución de inscripciones totales expresada en porcentaje según los diferentes talleres. Este índice se calcula considerando la suma de todas las inscripciones efectivas realizadas en los cinco talleres.
TABLA III

PORCENTAJE DE PARTICIPANTES POR NIVEL DE AVANCE DE LA CARRERA DE LOS TALLERES DE INGENIERÍA ELECTRÓNICA DE LA FACET-UNT

\begin{tabular}{|l|l|}
\hline $\begin{array}{l}\text { Nivel de avance de alumnos } \\
\text { de Ingeniería Electrónica }\end{array}$ & $\begin{array}{c}\text { Inscripciones } \\
\text { [\%] }\end{array}$ \\
\hline Primer Año & 4 \\
\hline Segundo Año & 10 \\
\hline Tercer Año & 35 \\
\hline Cuarto Año & 18 \\
\hline Quinto Año & 33 \\
\hline
\end{tabular}

TABLA IV

PORCENTAJE DE DISTRIBUCIÓN POR NIVEL DE AVANCE EN LA CARRERA DE INGENIERÍA ELECTRÓNICA DE LA FACET-UNT

\begin{tabular}{|l|l|l|l|l|l|}
\hline & \multicolumn{5}{|c|}{ Talleres } \\
\hline Nivel de avance & $\begin{array}{l}\text { PA } \\
{[\%]}\end{array}$ & $\begin{array}{l}\text { FO } \\
{[\%]}\end{array}$ & $\begin{array}{l}\text { PCB } \\
{[\%]}\end{array}$ & $\begin{array}{l}\text { PIC } \\
{[\%]}\end{array}$ & $\begin{array}{l}\text { FC } \\
{[\%]}\end{array}$ \\
\hline Primer Año & 0 & 8 & 0 & 10 & 0 \\
\hline Segundo Año & 21 & 8 & 0 & 10 & 0 \\
\hline Tercer Año & 36 & 38 & 36 & 30 & 0 \\
\hline Cuarto Año & 0 & 15 & 36 & 20 & 100 \\
\hline Quinto Año & 43 & 31 & 28 & 30 & 0 \\
\hline
\end{tabular}

TABLA V

PORCENTAJE DE DISTRIBUCIÓN POR CARRERA DE LOS PARTICIPANTES DE LOS TALLERES DE INGENIERÍA ELECTRÓNICA DE LA FACET-UNT

\begin{tabular}{|c|c|c|c|c|c|c|c|c|c|c|}
\hline & \multicolumn{9}{|c|}{ Talleres } \\
\hline Carrera & \multicolumn{1}{|l|}{ PA } & \multicolumn{1}{|c|}{ FO } & \multicolumn{1}{l|}{ PCB } & PIC & \multicolumn{2}{l|}{ FC } \\
\hline & $\%$ & Cant & $\%$ & Cant & $\%$ & Cant & $\%$ & Cant & $\%$ & Cant \\
\hline $\begin{array}{c}\text { Ingeniería } \\
\text { electrónica }\end{array}$ & 34 & 14 & 38 & 13 & 52 & 11 & 45 & 10 & 8 & 1 \\
\hline $\begin{array}{c}\text { Ingeniería } \\
\text { Biomédica }\end{array}$ & 32 & 13 & 24 & 8 & 19 & 4 & 36 & 8 & 23 & 3 \\
\hline $\begin{array}{c}\text { Ingeniería en } \\
\text { computación }\end{array}$ & 12 & 5 & 12 & 4 & 5 & 1 & 14 & 3 & 8 & 1 \\
\hline $\begin{array}{c}\text { Ingeniería } \\
\text { eléctrica }\end{array}$ & 7 & 3 & 3 & 1 & 0 & 0 & 0 & 0 & 8 & 1 \\
\hline $\begin{array}{c}\text { Otras } \\
\text { carreras }\end{array}$ & 5 & 2 & 0 & 0 & 5 & 1 & 0 & 0 & 31 & 4 \\
\hline Externas UA & 10 & 4 & 24 & 8 & 19 & 4 & 1 & 1 & 23 & 3 \\
\hline
\end{tabular}

TABLA VI

PORCENTAJE DE DISTRIBUCIÓN DE INSCRIPCIONES POR TALLERES

\begin{tabular}{|l|l|l|l|l|l|}
\cline { 2 - 6 } & \multicolumn{5}{|c|}{ Talleres } \\
\cline { 2 - 6 } & PA & FO & PCB & PIC & FC \\
\hline $\begin{array}{l}\text { Porcentaje de } \\
\text { alumnos [\%] }\end{array}$ & 31 & 26 & 16 & 17 & 10 \\
\hline
\end{tabular}

\section{DISCUSIÓN Y RESULTADOS}

Los talleres fueron concebidos para estudiantes de ingeniería electrónica de la FACET-UNT para impactar en los índices de permanencia, motivación, visibilidad y experiencia práctica mediante actividades extracurriculares.

Con el poco tiempo transcurrido de la primera edición de los cursos no se pueden inferir resultados cuantitativos con respecto a estos índices, pero sí se pueden inferir resultados cualitativos basados en la experiencia de esta primera edición.

Las encuestas implementadas a los alumnos participantes mostraron opiniones claramente positivas respecto nueva estrategia adoptada por la carrera, con lo que se puede inferir que se transita por el camino adecuado en cuanto a la visibilidad de la carrera. 
La actividad permitió captar el foco de los estudiantes de Ingeniería Electrónica, aspecto que se manifiesta en la Tabla II donde la mayoría de los estudiantes (38\%) que participaron de los talleres corresponden a estudiantes de Electrónica.

Por otro lado, los talleres alcanzaron todos los niveles de la carrera, desde primer año hasta quinto año. La Tabla III evidencia este aspecto y también permite afirmar que el $86 \%$ de los alumnos pertenece a tercero, cuarto y quinto año, siendo tercer año el curso de mayor participación.

La Tabla IV muestra un aspecto esperable en la participación de estudiantes de Electrónica: hay cursos que resultaron más atractivos que otros. El curso en el área de las ciencias complementarias en la formación integral del ingeniero (FC) registra el más bajo porcentaje de inscripción de estudiantes de la carrera. El taller más específico a la Ingeniería Electrónica (PCB) sólo tuvo estudiantes en el ciclo superior (tercer, cuarto y quinto año). Esto puede justificarse por el escaso contenido técnico electrónico en el primero y por la rigurosa especificidad del segundo que requiere del ciclo básico de formación para su entendimiento.

La Tabla $\mathrm{V}$ permite inferir que en todos los talleres excepto en el de formación complementaria fueron mayoría los estudiantes de Ingeniería Electrónica con respecto a estudiantes de otras carreras que también participaron. Se considera que los cursos de formación complementaria son tan importantes como los del área de electrónica, sobre todo en la nueva actualización plan de estudios basado en una educación por competencias donde las habilidades sociales también deben desarrollarse. Estas tablas dejan ver también la preferencia de los estudiantes de electrónica por el taller de Plataforma Arduino (PA) como primer lugar y el taller de Fibra Óptica (FO) en segundo lugar. Esta tendencia también se mantiene si se considera la totalidad de las carreras como se muestra en los datos de la Tabla VI.

\section{TRABAJOS FUTUROS}

La carrera pretende continuar trabajando en esta línea con futuras ediciones de los talleres para poder generar los suficientes datos para generar tendencias y estadísticas más robustas.

Se pretenden generar reportes sobre la trayectoria académica de los alumnos durante los dos primeros años que permita conocer:

a) si realiza alguna actividad académica,

b) si se inscribe en más de una carrera, qué actividades académicas realiza en cada una,

c) si abandona, conocer los motivos a través de la realización de encuestas dirigidas.

De los datos relevados se generarán indicadores que permitan monitorear la situación de los estudiantes.

Se espera la implementación de un sistema informatizado de seguimiento de graduados que forma parte del Plan de desarrollo institucional 2019-2025 de la UNT. De concretarse la carrera dispondrá de una base de datos confiable para generar políticas nuevas de seguimiento.

La carrera espera recabar información sobre las demandas formativas del sector socioproductivo, llevar información a los diferentes sectores del mercado laboral y organizar ciclo de charlas en la carrera a nivel de 3ro, 4to y 5to año con la visita de egresados destacados en los niveles socios productivos. Así mismo, se espera poder generar in programas de extensión conjunto entre docentes, investigadores y sector productivo que impliquen trabajos cortos de los estudiantes en las empresas o pasantías / prácticas profesionales.

\section{CONCLUSIONES}

Se ha presentado una estrategia académica para la carrera de Ingeniería Electrónica de la FACET-UNT, que permite fortalecer la permanencia, mejorar la visibilidad de la carrera tanto interna como externa a la UA, aumentar los índices de matriculación, mejorar la motivación de los estudiantes actuales de la carrera y finalmente, fomentar actividades extracurriculares acordes a estudiantes de todos los años del Plan de Estudio, como una manera de acercarlos al ejercicio de la profesión.

Con el poco tiempo transcurrido de la primera edición de los talleres no se puede concluir mejoras medibles significativas con respecto a los índices de permanencia y matriculación, pero sí se pueden afirmar mejoras cualitativas basados en la experiencia de esta primera edición.

Por otro lado, del análisis de las encuestas con opiniones de los estudiantes claramente positivos respecto a la nueva estrategia adoptada por la carrera, se puede concluir que se transita por el camino adecuado en aras de mejorar la visibilidad de la carrera.

Los resultados permiten concluir que se logró captar el foco de los estudiantes de Ingeniería Electrónica y, por otro lado, los talleres alcanzaron todos los niveles de la carrera, desde primer año hasta quinto año siendo mayoría en el ciclo superior.

\section{AGRADECIMIENTOS}

El presente trabajo se hizo posible gracias a los aportes materiales y cognitivos de la cátedra de "Diseño Lógico I" de la carrera de Ingeniería Electrónica, dictadas en la FACET-UNT.

La FACET-UNT aporto los recursos de infraestructura y logística necesarios para la difusión, publicidad y ejecución de los talleres.

\section{REFERENCIAS}

[1] J. Espinosa-Castro, J. Hernández-Lalinde, L. Castro, "Estrategias de permanencia universitaria," Scholarly Journals Archivos Venezolanos de Farmacologia y Terapéutica; Caracas Tomo 39 N. ${ }^{\circ}$ 1, pp. 88-97, Recibido: 12/12/2019 Aceptado: 16/02/2020

[2] Facultad de Ciencias Exactas y Tecnología de la Universidad Nacional deTucumán. URL: https://www.facet.unt.edu.ar/ ( Revisado setiembre 2020)

[3] Actualización del Plan de Estudios de Ingeniería Electrónica de la Universidad Nacional de Tucumán. Res HCS UNT 0305/2004 (2004)

[4] Plan Estratégico de Formación de Ingenieros 2012-2016. URL: https://confedi.org.ar/plan-estrategico-de-formacion-de-ingenieros2012-2016/ (Revisado setiembre 2020)

[5] P. Manzano, "Evaluación por competencias", Revista EDUCINADE, Ciudad Valles, México, vol 2, pp. 4-8, Diciembre 2019.

[6] Informe de autoevaluación institucional-2019. Universidad Nacional de Tucumán. URL: http:/www.unt.edu.ar/adjuntos/Informe-deAutoevaluacion-Institucional-2019.pdf

[7] Plan de desarrollo institucional 2019-2025 de la Universidad Nacional de Tucumán. URL: http://www.unt.edu.ar/adjuntos/PlanDesarrollo-2019.pdf

[8] Resoluciones de la FACET de aprobación de los talleres INGETRONICA. Res1276/19, Res1274/19 y Res1644/19 del HCD de la FACET 